\title{
URSETA - an interdisciplinary decision support tool for sustainable energy and mobility strategies
}

\author{
J. Forster ${ }^{1}$, T. Kaufmann ${ }^{2}$, N. Rab ${ }^{2}$ \& C. Winkler ${ }^{3}$ \\ ${ }^{I}$ Department of Spatial Planning, Vienna University of Technology, \\ Austria \\ ${ }^{2}$ Institute of Energy Systems and Electrical Drives, \\ Vienna University of Technology, Austria \\ ${ }^{3}$ Institute of Transportation, Vienna University of Technology, Austria
}

\begin{abstract}
The City of Vienna (Austria) follows a long-term initiative to become a Smart City. Efficient, affordable and low-carbon energy systems, as well as environmentfriendly transport systems are obligatory to provide a sustainable development of a city. URSETA (Urban Strategic Energy and Transportation Analyses) is a decision support tool which is able to communicate the dynamics of a city system and shows the potentials of implementing sustainable energy and transport techniques. Based on the Geographic Information System (GIS) data of two representative areas, the energy system including different types of energy carriers (natural gas, electrical, thermal) is analysed by an energy hub model. The transport behaviour of the city system is examined by implementing an integrated, dynamic transport and land-use model. The optimal energy and heat supply of the future city is computed by a mixed integer linear optimization problem. Various sustainable strategies with a time horizon of 10 and 20 years are evaluated in the URSETA model. The obtained results are implemented in a spatial 3D model. This model features dynamic information visualisation in a virtual reality environment and enables access for different types of stakeholders.

Keywords: decision support tool, 3D GIS spatial model, transport model, landuse model, energy hub model, energy supply, mixed integer linear optimization, sustainable strategies, urban strategies.
\end{abstract}




\section{Introduction}

"Imaging and building the future city is central to the future of humans in the regime of rapidly accelerating population group and declining resources, climatic and ecological change, and accelerating complexity of the global system." [1, p. 23] Achieving sustainable criterias for the future city requires an interdisciplinary work of various technical disciplines. This complicates the decision making in spatial planning processes. Thus visualisation models of energy and mobility systems gained rising interest in recent years. URSETA (Urban Strategic Energy and Transportation Analyses) combines both, a solid interdisciplinary technical foundation and a modern strategic spatial visualisation. Most notable visualisation models in this area include: The Chicago decarbonisation tool, ENUR and MARS.

The decarbonisation tool for Chicago was presented in 2011 by Daniel Segraves, Adrian Smith and Gordon Gill: "The creation of the multi-scale analytic model focusing on the embodied and expended carbon of urban systems allows for energy performance to be tracked across a wider region, encompassing multiple buildings and agents. [...] The primary function is an optimisation module programmed to determine the most efficient allocation of a given amount of resources towards growth or reconfiguration of the agents in question. [...] The model is designed to test and search the solution of possible retrofit and upgrade options for the group, arriving at the optimal use of these resources, to achieve the highest reduction of carbon consumption or the highest return on resource investment. It is a comprehensive decision process that incorporates carbon tracking, building energy analysis, design and planning optimisation, resource modelling and a 3-D graphic environment" [1, pp. 120-121].

The research project ENUR (Energy in the urban space) of the Department of Spatial Planning (Vienna University of Technology, 2011-2013) analyses the energy demand, supply, strategies and scenarios within the concept of energy and spatial planning. For this project a visualisation tool of the HLRS Stuttgart (High Performance Computing Center of the University of Stuttgart, Germany) was used for geometrical and visual display that can bee used for virtual reality walls too [2].

The simulation model MARS (Metropolitan Activity Relocation Simulator), developed by the Institute of Transportation at the Vienna University of Technology, is used to assess and to quantify the impacts of transport and/or land use policy instruments on economy, land use and environment. MARS is an integrated dynamic land use and transport model which simulates the effects of different transport- and land use planning policies over a time period of 30 years. The model is built using the Causal Loop Diagram (CLD) technique to improve transparency. This model was used in various cities including Leeds (UK), Ho Chi Minh City (Vietnam) and Mulhouse (France) [3]. 


\section{Motivation}

The Vienna University of Technology and the Wiener Stadtwerke Holding AG (Vienna Public Utilities Company) instituted a Doctorate College entitled "Urban Energy and Mobility Systems" (URBEM). Its goal is the analysis of scenarios and the development of strategies to achieve a sustainable, supply-secure, affordable and smart city. The theoretical model of this inter-disciplinary approach is applied to the mobility and energy systems of Vienna, Austria. The URBEM submodel URSETA (Urban Strategic Energy and Transportation Analyses) concerns heat and energy supply, energy supply networks and transport system as well as the visualisation of their output data in one single model.

The URSETA model focuses on fields that can be well regulated by the governance and have as well big potentials in decreasing $\mathrm{CO}_{2}$ emissions. In this regard the heat and energy supply model concerns the integration of renewable energies and decentral power generation (e.g. photovoltaic cells, ... ). The resulting strategies have a big influence on the energy supply network. Therefore the network needs to be analysed and optimized simultaneously from an electrical engineering point of view. The second big field of $\mathrm{CO}_{2}$ emittance is the transport system which promises huge potentials. In addition optimizing the transport system reduces noise and time effort and therefore enhances quality of living.

The interdependencies of these fields cause the necessity of an interdisciplinary modelling. For a successful implementation of strategies for a sustainable city the results of these submodels are merged and visualized, for a combined observation by professionals and all different types of stakeholders.

\section{Methodological framework for the URSETA model}

The URSETA model contains three submodels concerning energy and heat supply, mobility services and energy supply networks. As shown in Figure 1 the output data of the three submodels are merged and serve as the basement for the visualisation process. The three submodels have interdependencies and therefore need to be computed simultaneously. In particular the optimal investment strategies for energy supply depend on the constraints of the energy supply network. Moreover the economically optimal achievement of $\mathrm{CO}_{2}$ targets depends on suitable simultaneous reduction in the energy and mobility sector.

\subsection{Submodel 1: power and heat generation}

Many European energy service companies face the problem of finding longterm investment strategies for power and heat generation to meet new political and economic tasks including $\mathrm{CO}_{2}$ emission targets as well as rising economic inefficiency of gas power plants. Their investment strategies for electricity and heat generation have a huge impact on the sustainability of a city. Within the URSETA model we assumed that the energy service companies make cost-optimal investment decisions. 


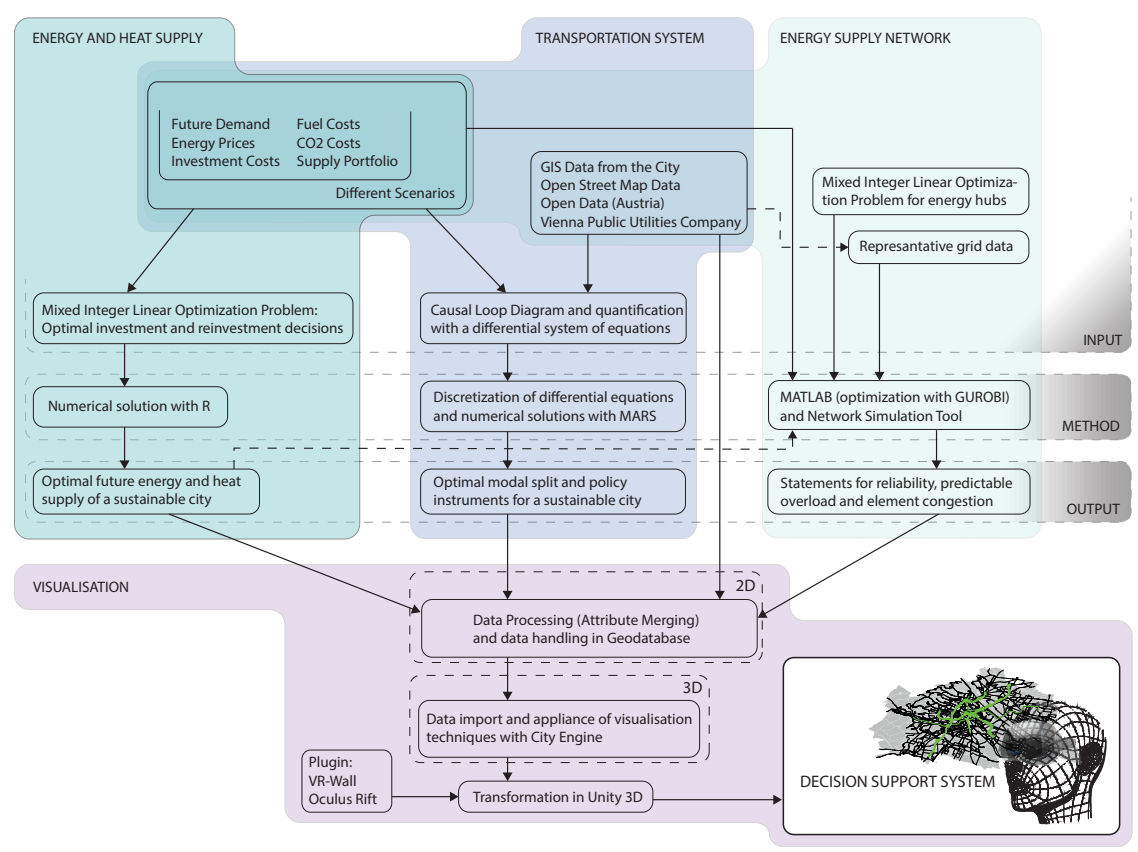

Figure 1: Methodological framework: submodels and interdependencies of the URSETA model.

These cost-optimal strategies can be computed as a solution a generation expansion planning (GEP) problem (see De Jonghe et al. [4] for an overview). A standard formulation of GEP is a mixed-integer linear optimization problem (MILP). Its objective function contains the discounted costs of electricity and heat generation for the planning periods and all discounted investment costs of new power plants. The input arguments include in particular different paths of energy demand, spot electricity and heat prices and feed-in-tariffs, fuel costs, investment costs and emission costs. Technological advancement is considered via learning curves for the investment and reinvestment costs. The modelling will allow for different types of power plants, e.g. plants with combined heat and power generation based on the algorithm by Lahdelma and Hakonen [5]. The control variable is the optimal investment (or alternative no investment) in new possible power plants. Thus the investment decision for every period are made such that the overall costs of investments and power and heat generations of every (future) period are minimal.

In the optimization problem the agent has the possibility to make investments $n_{i, t}$ in new power plants. If he does so in year $t$ for the $i$ th power plant then $n_{i, t}=1$, otherwise $n_{i, t}=0$. The agent tries to minimize the discounted future 
fixed costs $F_{i, t}$ (in Euro) for every period:

$$
\min _{n_{i, t}} \sum_{i, t} F_{i, t} n_{i, t}+O_{t}\left(n_{i, t}\right)
$$

with the operating costs $O_{t}\left(n_{i}\right)$ being a linear program for fixed $n_{i, t}$ :

$$
O_{t}\left(n_{i, t}\right):=\min _{g_{i, h}} \sum_{i, h} V_{i, h} g_{i, h} .
$$

Here $V_{i, h}$ and $g_{i, h}$ are the variable costs and generation for hour $h$ and power plant $i$. Additionally capacity constraints, demand constraints and specific operational constraints are needed.

There have been several attempts to incorporate the stochastic nature of the generation explanation planning problem. An overview of key contributions on generation expansion planning with specific emphasize on uncertainty modelling is given by Verderame et al. [6]. The deterministic formulation of the generation expansion problem with CHP technologies is generalized in the URSETA model as a two-stage stochastic optimization problem with complete recourse. The parameters of the second stage problem (demand, capacity, variable costs and spot electricity prices) are random variables on some probability space. In this formulation, at the first stage we have to make an investment decision $n_{i, t}$ before the realizations of the random variables are known.

\subsection{Submodel 2: mobility}

In order to compute future demand of public transport, as well as need for sustainable transport infrastructure for different scenarios we use an integrated land use and transport model. This submodel is based on differential equations characterizing relative changes of destination and transport selection based on place of residence and work as well as mobility supply. These differential equations are discretized and solved with VENSIM (Ventana Systems Inc.). The models in VENSIM are built using the Causal Loop Diagram (CLD) technique to improve transparency. Moreover predefined relations of the MARS model (see [3]) within VENSIM are used.

MARS is a strategic land use, transport interaction model capable of analysing policy combinations at the metropolitan level and assessing their impacts over a 30 year planning period. It includes a transport model which simulates the travel behaviour of the population related to their housing and workplace location, a housing development model, a household location choice model, a workplace development model, a workplace location choice model, as well as a fuel consumption and emission model. The sub-models are run iteratively over a 30 year time period. They are linked on the one hand by accessibility as output of the transport model and input into the land use model and on the other hand by the population and workplace distribution as output of the land use model and input 


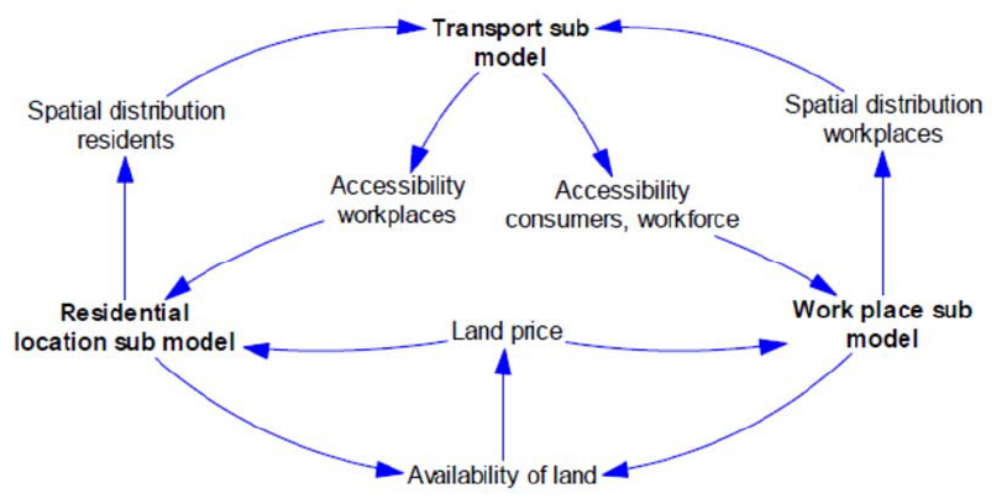

Figure 2: Submodel 2: main parts of the mobility model based on the MARS approach in VENSIM.

into the transport model. The model consists of a qualitative and a quantitative model.

The output of the submodel 2 shows the effects of urban policy measures and quantifies the potentials of changes in mobility behaviour. This information can be used to estimate the future demand of public transport, as well as need for sustainable transport infrastructure.

\subsection{Submodel 3: supply network}

Modelling and simulating a supply network for urban areas requires an approach with respect to the big amount of network data. Therefore modelling the hole supply network structure is not desirable. A breakdown to representative parts of the supply network is done [7]. Following this approach it is sufficient to model only the representative parts of an urban area for statements about the reliability, predictable overload and network congestions. This statements are applicable for the whole supply area of a power network.

An interdisciplinary system covering different power networks (e.g. natural gas, electric, thermal,...) is achieved by introducing an energy hub approach (Figure 3). The ideal model boundaries for an energy hub depend on the paths of development of the used scenarios. The optimal dispatch of the input energy carriers is achieved by solving a Mixed Integer Linear Optimization Problem (MILP) considering various storage techniques inside the energy hub. Simulation results of the optimization are used as an input for the supply network model simulation. So the desired results concerning reliability, predictable overload and network congestion can be achieved in an satisfying manner. 


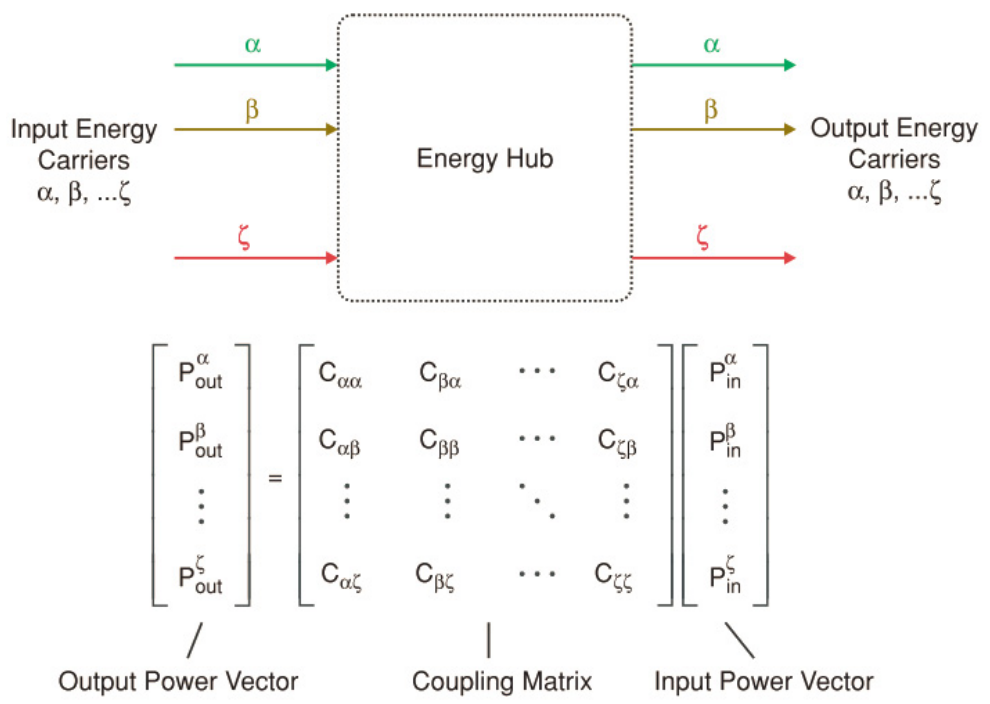

Figure 3: Modelling the transformation of power through an energy hub [8].

\subsection{Spatial visualisation}

The visualisation of city flows constitutes the main interface between researchers and stakeholders, the public and governance. It illustrates the research results and communicates new findings and ideas to a broad bandwidth of people. For this purpose the visualisation has to be easy understandable, simple to handle. It needs to cover different areas of expertise, several levels of detail as wells as different time horizons and areas. Especially the possibility of different scales of dimensions (full scale, detail,...) and options of views (top down-bottom up) has to be implemented. The data streams will be displayed dynamically in the 3D city environment which enables more potential for the information visualisation. Calculation and model based data of different disciplines and city overview data are 2-dimensional. Hence the main spatial visualisation model is based on static data. This requires abstraction for an adequate procedural illustration. For subsequent changes in the planning processes, parameters for controlling purposes have to be defined interdisciplinary.

Static 2-dimensional geo information system data (GIS) is the basis for the visualisation model. This data gives the opportunity to combine the storage of geometry as well as semantic additional information in connection. For spatial planning purposes this sort of data exists already for most areas worldwide. It can be expanded in rows and columns according to the users needs. For the transformation process from the second to the third dimension in space, the GIS based software City Engine (ESRI) and the NURBS (Non-uniform 
rational B-Splines) based CAD modelling software Rhinoceros 3D (Robert McNeel and Associates) is used. The whole information visualisation process is a transformation of abstract raw data into a visual form by using defined spatial boarders, markers and the assignment of graphic elements (visual mappings). A step further these defined graphical parameters can be macerated, changed or replaced (position, scale, colour, limits,...) through a process of perspectivechanges in the created visual structures.

The spatial visualisation process is handled with an application development system for video games (UNITY) and thus usable for diverse interactive users contemporaneously. Also dynamical and time-related data can be implemented via simulations. The game developer environment enables the visualisation of various aggregate states of objects. Looking at this implementation phase as a whole the main focus is the control of the visual achievement of objects, corresponding to the underlying data. This can be controlled via the predefined control parameters. In the visualisation process all detailed sub-steps and their possible connections, emerging during complex planning scenarios have to be considered. Also methodical approaches for typical spatial planning problems are preconceived. Afterwards the model becomes extended with analytic processes in spatial planning and graph and network based illustration methods. The combination of existing visualisation techniques and methods for the analysation process, plus their further development and redevelopment enable a systematic exploration and conceivable, space significant problems research of spatial city models. To simulate scenario specific procedures and explore their interdisciplinary independencies, concerning their predefined control parameters, relevant algorithms (Hill-Climbing, Roulette Wheel, Random Walk, A-StarAlgorithm, Genetic Algorithm,... ) are implemented.

All these add-ons create a full planning support system. This features interactive controlling input-mechanisms for the users and becomes, thanks gained insights, a decision support system for planning processes.

\subsection{Implementation and output}

To design strategies and analyse the future developments of a sustainable city, a digital city is needed. The concept of a digital city covers all types of computer designed models in virtual space. These city models comprise various scales (general overview to detailed descriptions) and form the basis for interdisciplinary analysis-methods.

The output of the URSETA model is a four dimensional model capable of simulations. Based on predefined control parameters various actors (including professionals, stakeholders, ... ) can change the model environment interactively via a video game based interface. Subsequently URSETA can be regarded as a decision support system which aims to help all phases of a planning process content wise and procedural (means while the process). To involve human cognitive and perception skills within a group, the use of a 3D virtual reality environment is beneficial. In particular with this environment the use of biological 


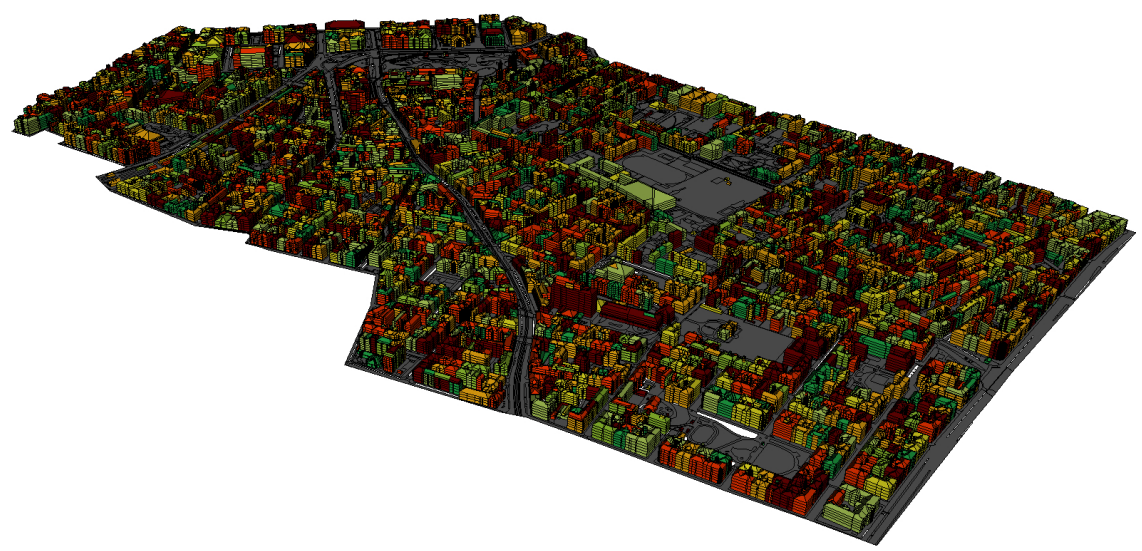

Figure 4: Heat demand (example data) for every single building within the worst case scenario in 2020 , for prototype area II.

adaptors (shutter classes, data glove, gesture control armband,...) for real time interaction can impart experiences close to reality. Moreover a free choice of perspectives is enabled which is essential for the acquisition and appraisement of spatial qualities. The best possible support of elementary spatial cognitive and conceptual processes is desired.

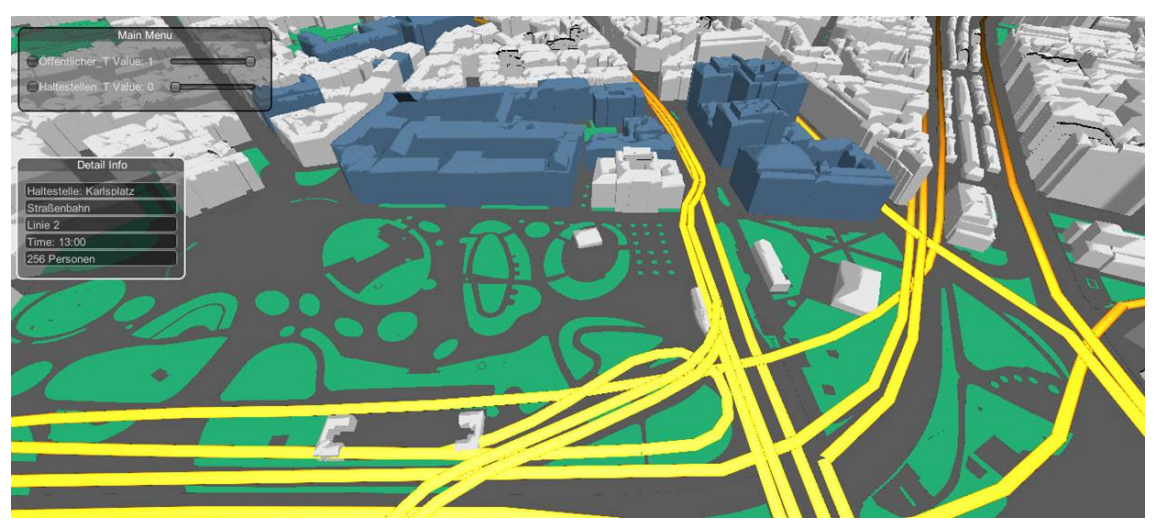

Figure 5: Visualization of public transport system.

Within the cooperation with the Wiener Stadtwerke Holding AG (Vienna Public Utilities Company) the URSETA model is applied to different city areas of Vienna, Austria. These areas are representatives for the present building stock of Vienna, in particular concerning building age, construction, electricity and heat demand. 
They also have a high level of mixed use of public and private buildings, diverse economic and environmental infrastructures as well as different transport modes sharing the surface densely. Figure 4 shows the URSETA model output for one prototype area (4th district of Vienna). The computed heat demand (example data) for every single building within one scenario (worst case scenario with no sustainable policy for 2020) is visualized.

\section{References}

[1] Weinstock, M., System City: Infrastructure and the space of flows. John Wiley \& Sons: West Sussex, 2013.

[2] Research project, ENUR, Energie im urbanen Raum. http://enur.project.tuwien.ac.at, 2014.

[3] Pfaffenbichler, P., The strategic, dynamic and integrated urban land use and transport model MARS. Ph.D. thesis, Vienna University of Technology, Research Center of Transport Planning and Traffic Engineering, 2003.

[4] De Jonghe, C., Hobbs, B. \& Belmans, R., Integrating short-term demand response into long-term investment planning. Cambridge Working Paper in Economics, 1132, pp. 3-4, 2011.

[5] Lahdelma, R. \& Hakonen, H., An efficient linear programming algorithm for combined heat and power production. European Journal of Operational Research, 148(1), pp. 141-151, 2003.

[6] Verderame, P.M., Elia, J.A., Li, J. \& Floudas, C.A., Planning and scheduling under uncertainty: A review across multiple sectors. Industrial \& Engineering Chemistry Research, 49(9), pp. 3993-4017, 2010.

[7] Florian Otto, Impact Assessment for a High Penetration of Distributed Generators in Medium and Low Voltage Grids. Master's thesis, TU Graz, IFEA, Graz, 2012.

[8] Geidl, M., Koeppel, G., Favre-Perrod, P., Klockl, B., Andersson, G. \& Frohlich, K., Energy hubs for the future. IEEE Power and Energy Magazine, 5(1), pp. 24-30, 2007. 